\title{
Erratum to"On Convolution surfaces in Euclidean spaces" Journal of Mathematical Sciences and Modelling, 1(2) (2018), 86-92
}

\author{
Selin Aydöner ${ }^{1}$ and Kadri Arslan ${ }^{1^{*}}$ \\ ${ }^{1}$ Department of Mathematics, Institute of Natural and Applied Science, Bursa Uludag University, Bursa, Turkey \\ * Corresponding author
}

\section{Article Info}

Keywords: Convolution of surfaces,

Flat surfaces, Gaussian curvature,

Minkowski sum, Second fundamental

form.

2010 AMS: 53C40, 53C42.

Received: 14 March 2019

Accepted: 10 April 2019

Available online: 20 April 2019

\section{Abstract}

In the present study we give some corrections for our paper which published in the first volume of this journal.

\section{Erratum to"On Convolution surfaces in Euclidean spaces"}

Page 89.

Theorem 3.2. Let $M \star N$ be a convolution surface of a paraboloid $M$ and a translation surface $N$ given with the parametrization (3.4). Then the Gaussian curvature of the convolution surface is

$$
K_{M \star N}=\frac{4 c f^{\prime \prime} g^{\prime \prime}}{\left(f^{\prime \prime}+2\right)\left(g^{\prime \prime}+2 c\right)\left(\left(f^{\prime}\right)^{2}+\left(g^{\prime}\right)^{2}+1\right)^{2}} .
$$

Proof. Let $M \star N$ be a convolution surface of a paraboloid $M$ and a translation surface $N$ given with the parametrization (3.4) For simplicity we define $z=x+y$. Then the tangent space of $M \star N$ is spanned by

$$
\begin{aligned}
z_{s} & =\frac{1}{2}\left(f^{\prime \prime}+2,0, f^{\prime}\left(f^{\prime \prime}+2\right)\right), \\
z_{t} & =\frac{1}{2 c}\left(0, g^{\prime \prime}+2 c, g^{\prime}\left(g^{\prime \prime}+2 c\right)\right) .
\end{aligned}
$$

Hence the coefficients of first and second fundamental forms of the convolution surface $M \star N$ are

$$
\begin{aligned}
& E=\left\langle z_{s}, z_{s}\right\rangle=\frac{1}{4}\left(\left(f^{\prime}\right)^{2}+1\right)\left(f^{\prime \prime}+2\right)^{2}, \\
& F=\left\langle z_{s}, z_{t}\right\rangle=\frac{f^{\prime} g^{\prime}}{4 c}\left(f^{\prime \prime}+2\right)\left(g^{\prime \prime}+2 c\right), \\
& G=\left\langle z_{t}, z_{t}\right\rangle=\frac{1}{4 c^{2}}\left(\left(g^{\prime}\right)^{2}+1\right)\left(g^{\prime \prime}+2 c\right)^{2},
\end{aligned}
$$

and

$$
e=\frac{\left\langle z_{s s}, z_{s} \times z_{t}\right\rangle}{\sqrt{E G-F^{2}}}=\frac{f^{\prime \prime}\left(g^{\prime \prime}+2 c\right)\left(f^{\prime \prime}+2\right)^{2}}{8 c \sqrt{E G-F^{2}}},
$$




$$
\begin{aligned}
& f=\frac{\left\langle z_{s t}, z_{s} \times z_{t}\right\rangle}{\sqrt{E G-F^{2}}}=0, \\
& e=\frac{\left\langle z_{t t}, z_{s} \times z_{t}\right\rangle}{\sqrt{E G-F^{2}}}=\frac{g^{\prime \prime}\left(f^{\prime \prime}+2\right)\left(g^{\prime \prime}+2 c\right)^{2}}{8 c^{2} \sqrt{E G-F^{2}}},
\end{aligned}
$$

respectively. By definition the Gaussian curvature of the convolution surface $M \star N$ is given by

$$
K_{M \star N}=\frac{e g-f^{2}}{E G-F^{2}} .
$$

So, substituting (3.5) and (3.6) into (3.7) after some calculation we get the result. $\square$

\section{Page 89.}

As a consequence of previous theorem one can get the following results.

Corollary 3.3. Let $M \star N$ be a convolution surface of a paraboloid $M$ and a translation surface (3.4). If the convolution $M \star N$ is a flat surface, then at least one of the following cases occur;

$$
f(s)=a_{1} s+a_{2}, \text { or } g(t)=b_{1} t+b_{2},
$$

where $a_{i}$ and $b_{j}$ are real constants.

Corollary 3.4. The convolution surface $M \star N$ given with the parametrization $f(s)=a_{1} s+a_{2}$ and $g(t)=b_{1} t+b_{2}$ is a part of a plane.

\section{Page 90.}

Finally,convolution surface $M \star N$ has the parametrization

$$
(x+y)(s, t)=\left(\frac{h^{\prime}+2 f f^{\prime}}{2 f^{\prime}} \cos t, \frac{h^{\prime}+2 f f^{\prime}}{2 c f^{\prime}} \sin t, \frac{\left(h^{\prime}\right)^{2}}{4 c\left(f^{\prime}\right)^{2}}\left(c \cos ^{2} t+\sin ^{2} t\right)+h(s)\right) .
$$

Theorem 3.5. Let $M \star N$ be a convolution surface of a paraboloid $M$ and a surface of revolution given with the parametrization (3.8). If $c=1$ then the convolution surface $M \star N$ also a surface of revolution with Gaussian curvature

$$
K_{M \star N}=\frac{\left(\varphi^{2}+h\right)^{\prime}\left\{\left(\varphi^{2}+h\right)^{\prime \prime}(\varphi+h)^{\prime}-\left(\varphi^{2}+h\right)^{\prime}(\varphi+h)^{\prime \prime}\right\}}{(\varphi+f)\left\{\left(\left(\varphi^{2}+h\right)^{\prime}\right)^{2}+\left((\varphi+h)^{\prime}\right)^{2}\right\}^{2}} ; f^{\prime} \neq 0,
$$

where $\varphi(s)=\frac{h^{\prime}(s)}{2 f^{\prime}(s)}$ is a real valued differentiable function different from 1 .

Proof. Similar to the proof of Theorem 3.2 we get the result.

Corollary 3.6. Let $M \star N$ be a convolution surface of a paraboloid $M$ with $c=1$ and a surface of revolution (3.8). If the convolution surface $M \star N$ is a flat surface, then it is either a plane or a surface of revolution satisfying

$$
\left(\varphi^{2}+h\right)^{\prime \prime}(\varphi+h)^{\prime}-\left(\varphi^{2}+h\right)^{\prime}(\varphi+h)^{\prime \prime}=0
$$

Proof. If $M \star N$ is a flat surface, then

$$
\left(\varphi^{2}+h\right)^{\prime}\left\{\left(\varphi^{2}+h\right)^{\prime \prime}(\varphi+h)^{\prime}-\left(\varphi^{2}+h\right)^{\prime}(\varphi+h)^{\prime \prime}\right\}=0
$$

holds. So, we have two possible cases; $\varphi^{2}+h=$ const. , or $\left(\varphi^{2}+h\right)^{\prime \prime}(\varphi+h)^{\prime}-\left(\varphi^{2}+h\right)^{\prime}(\varphi+h)^{\prime \prime}=0$. For the first case $M \star N$ is a part of a plane $\square$.

\section{Page 90.}

Omit the Equation 3.14.

Finally, the sum $M \star N$ has the parametrization

$$
(x+y)(s, t)=\left(\begin{array}{c}
\left(\frac{2 t p(s)-z^{\prime}(s)}{2 t}\right) \sin s+\left(p^{\prime}(s)+t\right) \cos s \\
\left(\frac{z^{\prime}(s)-2 c t p(s)}{2 c t}\right) \cos s+\left(p^{\prime}(s)+t\right) \sin s \\
z(s)+\left(\frac{z^{\prime}(s)^{2}}{4 c t^{2}}\right)\left(c \sin ^{2} s+\cos ^{2} s\right)
\end{array}\right), t \neq 0 .
$$

Page 91. 
Theorem 3.7. Let $M \star N$ be a convolution surface of a paraboloid $M$ with $c=1$ and a right helicoid $N$ given with the parametrization (3.17). Then the Gaussian curvature of the convolution surface is

$$
K_{M \star N}=-\frac{\psi^{\prime \prime}\left\{\left(\psi^{\prime}(t-k) t+\psi\left(\psi \psi^{\prime}+k\right)\right\}(k-t)-\left\{\psi \psi^{\prime}+\left(\psi^{\prime}\right)^{2}(k-t)+k\right\}^{2}\right.}{\left\{\left(\psi^{\prime}\right)^{2}(k-t)^{2}+\left(\psi \psi^{\prime}+k\right)^{2}+\left(\psi \psi^{\prime}+t\right)^{2}\right\}^{2}} ; t \neq 0,
$$

where

$$
\psi(t)=\frac{-k}{2 t},
$$

is a real valued function.

Proof. Similar to the proof of Theorem 3.2 we get the result. 